\title{
Tumor infiltrating lymphocytes in malignant melanoma - allies or foes?
}

\author{
Vladimir Zidlika,b, Michala Bezdekovac, Svetlana Brychtova ${ }^{\mathrm{cd}}$
}

This is an overview of current problematics regarding the role of tumor infiltrating lymphocytes (TILs) in malignant melanomas. Various and often conflicting data have been published, correlating tumor type, stage, prognosis, as well as sex and age of patients. This is partly due to heterogeneity in scaling systems and unstandardized TILs grading but also due to changes of tumor-host interactions. Melanomas are an immunologically heterogeneous group with variability of TILs, where distinct gene expression patterns were found in tumors with absent, and/or non- brisk TIL grade versus brisk TIL grade. However, the presence of TILs alone appears to be inadequate for implicating them as immunologically functional. Further characterisation of TIL phenotype and function is warranted. This especially concerns, evaluation of TILs of the suppressor phenotype but rather than as a prognostic factor, more for prediction of targeted immunotherapy.

Key words: melanoma, tumor infiltrating lymphocytes, regulatory lymphocytes, immune check point

Received: May 15, 2019; Revised: 29.8.2019; Accepted: September 11, 2019; Available online: October 24, 2019

https://doi.org/10.5507/bp.2019.048

(c) 2020 The Authors; https://creativecommons.org/licenses/by/4.0/

${ }^{a}$ Department of Pathology, University Hospital Ostrava, Czech Republic

${ }^{b}$ Department of Pathology, CGB Laboratory, Ostrava, Czech Republic

'Institute of Clinical and Molecular Pathology, University Hospital Olomouc, Olomouc, Czech Republic

dInstitute of Clinical and Molecular Pathology, Faculty of Medicine and Dentistry, Palacky University Olomouc, Czech Republic

Corresponding author: Svetlana Brychtova, e-mail: svetlana.brychtova@seznam.cz

\section{INTRODUCTION}

Tumor growth is a complex process that involves numbers of interactions between tumor cells and immune host system through multiple cellular and molecular factors in the tumor specific microenvironment. Many genetic and epigenetic alterations in cancer cells provide a diverse set of antigens ${ }^{1}$, which are recognized by the immune system. An effective cancer immune surveillance system is able to destroy premalignant and malignant cells, but its avoidance can be considered as a hallmark of cancer. The term "immunoediting" has been proposed to describe the events when the immune system interacts with malignant cells during the course of cancer ${ }^{2}$. Interaction between tumor and immune cells can crucially determine the outcome of the disease. Anticancer immune response is a very complex process, where $\mathrm{T}$ lymphocytes which are able to recognize specific neoantigens, play a key role. Tumor infiltrating lymphocytes (TILs) are lymphocytes in close association with cancer cells and have infiltrated and disrupted nests of malignant cells. They infiltrate the central areas of tumors as well as their edges in a neighbourhood with healthy non-tumorous tissue. As they interact most closely with the cancer cells, they are likely to more accurately reflect tumor-host interactions. Increased density of TILs is recognized as a sign of efficient antitumor immunity and generally associated with a better outcome. There are still many obscurities and questions deserving clarification.

Melanoma is considered an extremely immunogenic neoplasm. This is partly due to its higher genomic instability leading to formation of many neoantigens which are recognized by immune cells. Activation of the immune system is observed even in dysplastic nevi, which are taken as melanoma precursors. We know that the immune response cannot directly prevent melanoma formation; however, it can make a difference to the disease outcome ${ }^{3}$. The melanoma immunogenity is reflected by the density of lymphocytic infiltrate surrounding the malignant cells, as well as by the relatively high grade of melanoma spontaneous regressions. What is interesting is that despite this regression, which is an obvious result of local elimination of malignant cells by immune cytotoxicity, in many cases there was metastatic spread of tumor cell, which is a prognostically negative feature ${ }^{4}$. Better understanding of an effective antitumor immune response in patients with melanoma is desirable for assessment of a disease prognosis and prediction, as improved survival has been shown by blocking mechanisms that are responsible for immune down regulation ${ }^{5,6}$.

\section{Quantification of TILs in melanoma}

One of the most frequently used scoring system is Clark's method grading TILs as absent, non-brisk, or brisk $^{7,8}$. Other studies grades TILs infiltrate as a range of 0 to 3 or even only as absent or present ${ }^{9,10}$. Heterogeneity in scaling system and a lack of standardization of TILs grading method contributes to limitation and diversity of results in the current literature on the prognostic value of TILs, along with the heterogeneity of patients' selection criteria and often small sample sizes. This may explain the often controversial data regarding variability in numbers of TILs according a melanoma type and its outcome. 


\section{TILs and the age and sex of patients}

Age is an independent negative predictor of melanoma survival. Multiple factors may contribute to this observation including decline in host antitumor immune response with aging. It is not so surprising that a higher TIL grade has been found to be more common in younger patiens ${ }^{11}$. On the other hand, once brisk TIL grade in old patients was found, it was associated with prolonged melanomaspecific survival ${ }^{12}$. Evaluation of TILs may so have greater prognostic value in patients above 45 years ${ }^{12}$.

It has been documented that sex also affects clinical outcomes of melanomas, as women tend to exhibit a more favourable melanoma-specific prognosis in comparison to men. Women have also been reported to have better outcomes in overall survival (OS) and a lower tendency for metastasis. A relationship between sex and sentinel lymph node (SNL) positivity has been inconsistently reported. The precise mechanism for this sex-specific prognosis still remains unclear, it is probable that varying patterns of immunity might be involved. SNL metastases were associated with brisk TILs among men but not in women. Also among men prolonged OS was associated with presence of brisk TILs. However, no association between TIL status and OS among women has been found ${ }^{13}$.

However, there are also opposite data, where age difference and male to female ration did not differ between different grades of TILs (ref. ${ }^{3}$ ).

\section{TILs and melanoma type}

Concerning melanoma type, there is not full consensus regarding relation between histological type and intensity of TILs. Whereas some authors found no differences, especially between more frequent melanoma types as acral lentiginous, lentigo maligna, nodular, superficial spreading ${ }^{3}$, others documented their significant decrease in some types as are desmoplastic and nodular melanomas $^{12}$. These melanomas have a less favourable prognosis, based on their apparently aggressive clinical behaviour.

\section{TILs and worse pathologic characteristic}

Various, often conflicting data have been published regarding worse melanoma histopathologic features such as ulceration, increased mitotic activity or formation of satellite foci. Generally, absence of infiltrates was found in tumors with increased mitotic activity and ulceration ${ }^{14}$. Significant correlation also exists with diminished rate of microsatellitosis and increased numbers of TILs (ref. ${ }^{3}$ ).

\section{TILs and melanoma stage and prognosis}

Melanomas with brisk density are more frequently associated with better prognosis than tumors in which lymphocytic infiltrate was absent or low. But only brisk TILs have been shown to improve recurrence-free survival and overall survival compared to non-brisk or absent metastatic melanomas, where TILs are low or absent ${ }^{12,15}$. Several studies found that TILs response as a major predictor of sentinel lymph node metastasis, whereas metastasis decreased with increasing incidence of TILs (ref. ${ }^{3,16}$ ). However, others published that TILs did not predict sur- vival $^{17}$ and there is no association of their presence and improved melanoma prognosis ${ }^{11,17-19}$.

It is assumed that one of major determinants of immune cell infiltration is the stage of disease where host immune answer decreases with increasing tumor thickness ${ }^{20}$. Consistent results are published for advanced T4 melanoma. In T1-T3 stages, however, there are diverse data where even T3 tumors may have higher TILs, while in $\mathrm{T} 1$ or T 2 melanomas their density may be $10 \mathrm{w}^{21}$. It also has been described that the distribution of TILs is heterogenous, and in more advanced stages they become more accumulated at the peripheral areas associated with invasiveness. Heterogeneity of TILs distribution should be taken in consideration as it seems to support tumor progression $^{21}$. The paradox that presence of TILs does not always correlate with improved prognosis may be explained by the presence of different lymphocyte subtypes $^{22}$. Hence, the role of TIL has been studied with an increased interest.

\section{The role of CD8+T lymphocytes}

CD8+T cells belong to main subsets of T cells that constitutively mediate an effective antitumor response. Their transfer to patients with melanomas has been shown to be associated with beneficent therapeutic effect. While CD8+T cells can produce Th1 (T helper type 1) cytokines when activated, their primary functions is production of cytotoxic proteins, mainly perforin and granzyme, which are secreted at the point of the immunologic synapse - the place of contact with the target cell. Then specific killing without bystander cellular damage is a result of the process. Perforin is a protein disrupting cellular membrane and it also facilitates the ability of granzymes to induce apoptosis in the target cell ${ }^{23}$.

Antigens that are expressed on cancer cells and recognized by T lymphocytes are used in tumor-specific immunotherapy. However, the effectiveness of currently available therapeutic strategies is limited, as only about $5 \%$ of vaccinated patients with metastatic melanoma exhibited a complete or partial clinical response, whereas additional 10\% showed limited evidence of melanoma regression without clear clinical benefit ${ }^{24}$.

\section{The role of CD4+ T lymphocytes}

Important role for activation and maintenance of immune response against tumor cells also belongs to CD4+ T lymphocytes. Although these cells have ability to eliminate tumor cells in the absence of cytotoxic lymphocytes, more often both cell types are required for tumor rejection $^{25}$. As the main effector mechanism of antitumor immune response is direct cells lysis through the major histocompatibility complex (MHC) class I recognizing CD8+ CTL (cytotoxic lymphocytes), the key role of CD4+ $\mathrm{T}$ cells is in the activation of CTL. Based on their cytokine profile, CD4+ T-cell response can be sub-classified into different types. Th1 cells, which typically produce interleukin-2 (IL-2), lymphotoxin $\alpha$ (LT $\alpha$ ) and interferon- $\gamma$ $(\mathrm{IFN} \gamma)$ are able to induce macrophage activation, and also proliferation and activation of $\mathrm{CD} 8+\mathrm{T}$ cells ${ }^{26}$. Th 1 
response is generally correlated with a better cellular immune activation ${ }^{27}$. In contrast, Th2 (T helper type 2) cells produce IL-4, IL-5, IL-10 and IL-13 and tend to elicit humoral immunity. In some reports, Th2 and their cytokines have been shown to down regulate antitumor activity ${ }^{28}$. A shift from a Th1 to a Th2 cytokine profile is considered to be a major contributor to the failure of $\mathrm{T}$ cell mediated immunity. On the contrary, there are many opposite data that Th2 specific clones have been demonstrated to strong anti-tumor activity. The mechanisms how Th 2 cells destroy tumors are not fully understood, but it may be through the activation of innate immune response, such as eosinophils and macrophages which in turn secrete superoxide and nitric oxide. Effective anti-tumor immunity seems to be a result of balance cooperation between Th1 and Th2 cell types.

\section{Melanomas with present TILs represent an immunologically heterogenous group}

A gene expression analysis identified immunologic heterogeneity among melanomas depending on the intensity of lymphocytic infiltrate. Melanomas with absent and non-brisk TIL grades had the most similar immunoregulatory gene expression patterns and were immunologically distinguishable from tumors with brisk TIL grade. This group of melanomas had gene expression profile associated with $\mathrm{T}$ lymphocyte activation which encompass $\mathrm{T}$ helper cell differentiation, $\mathrm{T}$ cell receptor, dendritic cell maturation, T cell co-stimulation (CD28, CD5), cytokine signalling (IL-2), and other signalling pathways like interferon, Toll-like receptor and JAK/STAT (Janus kinase/ Signal Transducers and Activators of Transcription) signalling. Moreover, immune checkpoint regulators such as PD-1 (Programmed cell death 1) and CTLA4 (Cytotoxic T-Lymphocyte Associated Protein 4) were more inhibited. It could be emphasized that melanoma with brisk TILs represent a distinct immunologic entity ${ }^{12}$.

\section{Adverse effect of TILs}

Despite the well established immunogenicity of many melanomas, it is obvious that immune answer often fails to regulate and/or inhibit tumor progression. Several local factors have been described to be responsible for attenuation of effective immune response, and an immunosuppressive microenvironment is created. In this way, immune cells may not only help cancer cells to escape immune surveillance, they are able even to support tumor progression.

\section{T regulatory lymphocytes (Tregs)}

Tregs are CD25+CD4+ T lymphocytes, they are considered to be one of the main regulatory cell types which inhibit and effector functions by dampening the T-cellmediate immune response against the tumor cells ${ }^{5,29-31}$. The transcription factor FOXP3 (forkhead box P3) plays a key role in CD4+ CD25+ regulatory T cell function and differentiation and represents a specific marker for these cells $^{32}$. FOXP3 is a member of the forkhead or winged helix family of transcription factors ${ }^{33,34}$. Tregs can be recruited in the periphery from conventional CD4+ helpers (adaptive/induced cells) where T lymphocytes infiltrating the area of a tumor may be compromised or may adversely adapt to the suppressive environment to promote growth instead of regression ${ }^{31}$. Their majority is generated during normal process of maturation in the thymus. Recent reports suggest a role for TGF- $\beta$ (transforming growth factor $\beta$ ) in generation of Tregs from CD4+CD25- precursors ${ }^{35}$.

Tregs account for only $5-10 \%$ of CD4+ cells, their activation is associated with inhibition of cytotoxic lymphocytes and NK (Natural Killer) cells ${ }^{36}$. However, the precise role of Tregs in cancer development and progression is not clear. In many studies Tregs were proved to promote growth of many cancers. They are responsible for immune tolerance against tumor antigens by dampening the $\mathrm{T}$ cell mediated immune response, which enable malignant cells to evade the immune destruction. Mechanism of their immunosuppression is dependent on a mutual intercellular contact which leads via granzyme and/or perforin dependent pathway to inhibition of $\mathrm{T}$ cell proliferation. Also in malignant melanoma recent studies have documented that the presence of Foxp3+ Tregs in the microenvironment may significantly contribute to the immune resistance. This is supported by results of both experimental animal melanoma models as well as patient studies where high numbers of circulating Tregs were associated with rapid progression of the disease. The presence of FOXP3 lymphocytes in primary cutaneous melanomas were associated with a higher frequency of metastases in the sentinel lymph node ${ }^{37,38}$. FOXP3 expression in melanomas so seems to be associated with worse overall survival and FOXP3+ Tregs are thought to be predictive of patient survival as a marker of early metastatic spread or recurrence of the disease ${ }^{22,39-42}$. Moreover, therapeutic inhibition of Tregs has been proved to impair their immunosuppressive effect, which is associated with better outcome of the disease ${ }^{43}$.

Higher densities of Treg were observed in more advanced stages of melanoma in a vertical growth phase and in metastatic melanomas ${ }^{22,39,42}$. In more advanced stages of melanomas the most pronounced changes in CD3+/Treg ratio in behalf of Tregs have been also found. What is interesting that higher accumulation of Tregs was predominantly at the periphery of tumors, at the front line in the neighbourhood of benign tissue. This finding, indicating that advanced tumors create an immunosuppressive microenvironment, may explain their resistance against immune destruction ${ }^{21,44}$. Moreover, heterogeneity of lymphocytic infiltrate should be taken into consideration, where immunosuppression in the front line may promote melanoma progression. Surprisingly, some T4 stages of melanoma exhibited low Treg density which may be a feature of a severe failure of immune system, and be another feature of higher aggressiveness of the tumor as a presence of Tregs reflects at least partially preserved immune functionality. Similar changes were also for example described in colorectal carcinomas ${ }^{45}$.

\section{Immune Checkpoints}

Immune checkpoint molecules refer to a group of immune receptors that upon engagement with their ligand 
transmit inhibitory signals suppressing effector function. In this way cancer may evade anti-tumor imunity ${ }^{46}$. Programmed cell death signalling pathway (PD-1/PD-L1) (Programmed death-ligand 1) has become one of the most discussed inhibition pathways. PD-1 is expressed on many immune cells; among them $\mathrm{T}$ lymphocytes belong to the most important. PD-1 is activated by its ligands PD-L1, also known as B7-Hi protein and PD-L2 (Programmed death-ligand 2) ( ref. $^{47,48}$ ).

The engagement of PD-1 by its ligands induces apoptosis or exhaustion in activated T cells and some cytokines production, such as IFN- $\gamma$ and IL-2. The result is lymphocyte deletion and establishment of immunological tolerance ${ }^{49}$. Compared to PD-L2 that can be found only in activated dendritic cells and macrophages, PD-L1 is expressed by $\mathrm{T}$ and $\mathrm{B}$ cells, macrophages and often on many tumors including melanoma ${ }^{50,51}$.

Once TILs recognize tumors they start to produce IFN- $\gamma$ and other pro-inflammatory cytokines up-regulating PD-L1 expression. PD-L1, in turn, may ligate PD-1 antigen on lymphocytes inducing their down regulation. This adaptive PD-L1 expression in the melanoma microenvironment has been associated with an improved prognosis. It also may be a predictor of anti-PD1/PD-L1 targeted therapy response.

On the other hand, constitutive PD-1 expression by tumor specific $T$ cells was associated with the induction of inhibitory receptors expression followed by an impairment of $\mathrm{T}$ cell functions, tumor cells may then escape upon ligation to PD-L1. This is for example accompanied by loss of PTEN (Phosphatase and tensin homologue) with consequent activation of PI3K (Phosphoinositide 3-kinase) pathway. Constitutive PD-1 expression so constitutes a form of immune adaptation and tumor tolerance.

Considering up-to now published data, there is an ambiguous and not fully understood role of PD-1 in creation of efficient or ineffective $\mathrm{T}$ lymphocyte responses ${ }^{52}$. In spite of an indisputable inhibitory signalling upon ligation with its ligands, it is now clear that PD-1 expression is a first marker of $\mathrm{T}$ cell activation allowing the identification of the tumor reactive CD8+ $\mathrm{T}$ cell population in melanoma tumors ${ }^{53,54}$. Moreover, it is a sign of high avidity of cytotoxic lymphocytes to specific neoantigens, the level of PD-1 expression so reflects functionality of specific T cells.

The complex regulation of PD-1 expression unequivocally demonstrates that PD-1 expression status alone cannot distinguish between exhausted and activated $T$ cells that are the result of distinct genetic and epigenetic programs dictated by T-cell receptor signalling strength and microenvironment. It also explains the fact that, despite PD-1 undisputed clinical effectiveness compared to chemo- or radiotherapy, anti-PD-1 monotherapy remains inefficient in more than $60 \%$ of cancer patients ${ }^{55}$.

There are studies highlighting that PD-L1 expression may depend on melanoma subtype. Melanomas with high UCV radiation exposure (high cumulative sun damage) had the highest expression of PD-L1; it reflects high mutational load in these tumors and provide a possible ex- planation that these patients had high rates of responses to anti-PD-1 monotherapy. By contrast, uveal, acral and mucosal melanomas demonstrate the lowest TIL densities and PD-L1 positivity which may be due to the fact they have the least genomic instability. These melanoma subtypes also exhibit low response rates to anti-PD-1 monotherapy.

Furthermore, PD-1 expression seems to be dependent on tumor thickness with higher density in T3 and T4 melanomas. Also distribution of these lymphocytes is not homogenous, they tend to be increased at the tumor edges ${ }^{21}$. Peripheral areas, where malignant cells meet neighbour microenvironment, seem to be more critical for the fate of the lesion.

\section{CONCLUSION}

Melanoma represents immunologically heterogeneous group with variability of TILs predominantly in dependence on the type and stage of the disease with inconsistent results regarding outcome of the disease. The presence of TILs alone is not enough to implicate them as immunologically functional. In light of recent studies interested in the possible benefit of TILs to determine therapeutic outcome, further characterisation of TIL phenotype and their function seems to be important. Especially, evaluation of TILs focusing on suppressor phenotype is essential, but rather than as a prognostic factor, more for prediction of targeted immunotherapy. However, still some degree of ambiguity exists, which will require further investigation.

\section{ABBREVIATIONS}

TILs, Tumor infiltrating lymphocytes; OS, Overall survival; SNL, Sentinel lymph node; Th1, T helper type 1; MHC, Major histocompatibility complex; CTL, Cytotoxic lymphocytes; IL-2, Interleukin-2; LT $\alpha$, Lymphotoxin $\alpha$; IFN $\gamma$, Interferon- $\gamma$; Th2, T helper type 2; JAK/STAT, Janus kinase/Signal Transducers and Activators of Transcription; PD-1, Programmed cell death 1; CTLA4, Cytotoxic T-Lymphocyte Associated Protein 4; Tregs, T regulatory lymphocytes; FOXP3, Forkhead box P3; TGF- $\beta$, Transforming growth factor $\beta$; NK, Natural Killer; PD-L1, Programmed death-ligand 1; PD-L2, Programmed death-ligand 2; PTEN Phosphatase and tensin homologue; PI3K, Phosphoinositide 3-kinase.

\section{Search strategy and selection criteria}

Our research strategy was focused on evaluating of studies on the role of tumor infiltrating T lymphocytes in cutaneous malignant melanoma progression. In particular, we aimed at $\mathrm{T}$ regulatory lymphocytes and immune checkpoint molecules PD-1/PD-L1. Scientific articles from 1989 to 2019 were searched using the PubMed, Web of Science databases, and Researchgate. All searches were up to date as of March 2019. The searched terms 
included melanoma, tumor infiltrating lymphocytes, $\mathrm{T}$ regulatory lymphocytes, immune checkpoints, stage, prognosis. Czech and English language papers were reviewed.

Acknowledgement: This publication was supported by the grant of Internal Grant Agency, Palacký University, Olomouc, Czech Republic no. IGA UP LF_2019_004.

Author contributions: VZ, SB: literature search and manuscript writing, MB: critical reading and manuscript revision.

Conflict of interest statement: The authors declare no conflict of interest.

\section{REFERENCES}

1. Ohkura N, Sakaguchi S. Regulatory T cells: roles of T cell receptor for their development and function. Semin Immunopatho 2010;32(2):95-106

2. Gooden MJ, de Bock GH, Leffers N, Daemen T, Nijman HW. The prognostic influence of tumour-infiltrating lymphocytes in cancer: a systematic review with meta-analysis. Br J Cancer 2011;105(1):93-103.

3. Ghanadan A, Ehsani AH, Farahmand AM, Mirzaei M. Tumor Infiltrating Lymphocytes in Different Stages of Malignant Melanoma and Correlation with Tumor Stage and Other Prognostic Factors: A Retrospective Multicenter Study Middle East J Cancer 2017;8(4):20712.

4. Ribero S, Moscarella E, Ferrara G, Piana S, Argenziano G, Longo C. Regression in cutaneous melanoma: a comprehensive review from diagnosis to prognosis. J Eur Acad Dermatol Venereol 2016;30(12):2030-7.

5. Gerber AL, Münst A, Schlapbach C, Shafighi M, Kiermeir D, Hüsler $\mathrm{R}$, Hunger RE. High expression of FOXP3 in primary melanoma is associated with tumour progression. Br J Dermatol 2014;170(1):103-9.

6. Harter PN, Bernatz S, Scholz A, Zeiner PS, Zinke J, Kiyose M, Blasel S, Beschorner R, Senft C, Bender B, Ronellenfitsch MW, Wikman $\mathrm{H}$, Glatzel M, Meinhardt M, Juratli TA, Steinbach JP, Plate $\mathrm{KH}$, Wischhusen J, Weide B, Mittelbronn M. Distribution and prognostic relevance of tumor-infiltrating lymphocytes (TILs) and PD-1/ PD-L1 immune checkpoints in human brain metastases. Oncotarget 2015;6(38):40836-49.

7. Clark WH Jr, Elder DE, Guerry D 4th, Braitman LE, Trock BJ, Schultz $D$, Synnestvedt M, Halpern AC. Model predicting survival in stage I melanoma based on tumor progression. J Natl Cancer Inst 1989;81(24):1893-904.

8. Dundr $P$, Němejcová $K$, Bártů $M$, Tichá I, Jakša R. Hodnocení zánětlivé infiltrace (tumor infiltrujících lymfocytů - TIL) u maligního melanomu. Cesk Patol 2018;54(1):27-32.

9. Azimi F, Scolyer RA, Rumcheva P, Moncrieff M, Murali R, McCarthy SW, Saw RP, Thompson JF. Tumor-infiltrating lymphocyte grade is an independent predictor of sentinel lymph node status and survival in patients with cutaneous melanoma. J Clin Oncol 2012;30(21):267883.

10. Tuthill RJ, Unger JM, Liu PY, Flaherty LE, Sondak VK. Risk assessment in localized primary cutaneous melanoma: a Southwest Oncology Group study evaluating nine factors and a test of the Clark logistic regression prediction model. Am J Clin Pathol 2002;118(4):504-11.

11. Mandalà $M$, Imberti GL, Piazzalunga $D$, Belfiglio $M$, Labianca $R$, Barberis M, Marchesi L, Poletti P, Bonomi L, Novellino L, Di Biagio K, Milesi A, Guerra U, Tondini C. Clinical and histopathological risk factors to predict sentinel lymph node positivity, disease-free and overall survival in clinical stages I-II AJCC skin melanoma: outcome analysis from a single-institution prospectively collected database. Eur J Cancer 2009;45(14):2537-45.

12. Weiss SA, Han SW, Lui K, Tchack J, Shapiro R, Berman R, Zhong J, Krogsgaard M, Osman I, Darvishian F. Immunologic heterogeneity of tumor-infiltrating lymphocyte composition in primary melanoma. Hum Pathol 2016;57:116-25.

13. Sinnamon AJ, Sharon CE, Song Y, Neuwirth MG, Elder DE, Xu X, Chu
EY, Ming ME, Fraker DL, Gimotty PA, Karakousis GC. The prognostic significance of tumor-infiltrating lymphocytes for primary melanoma varies by sex. J Am Acad Dermatol 2018;79(2):245-51.

14. Thomas NE, Busam KJ, From L, Kricker A, Armstrong BK, AntonCulver H, Gruber SB, Gallagher RP, Zanetti R, Rosso S, Dwyer T, Venn A, Kanetsky PA, Groben PA, Hao H, Orlow I, Reiner AS, Luo L, Paine S, Ollila DW, Wilcox H, Begg CB, Berwick M. Tumor-infiltrating lymphocyte grade in primary melanomas is independently associated with melanoma-specific survival in the population-based genes, environment and melanoma study. J Clin Oncol 2013;31(33):4252-9.

15. Fu Q, Chen N, Ge CH, Li R, Li Z. Prognostic value of tumor-infiltrating lymphocytes in melanoma: a systematic review and meta-analysis. Oncolmmunology 2019;3;8(7):1593806. doi: 10.1080/2162402X.20 19.1593806

16. Lee N, Zakka LR, Mihm MC Jr, Schatton T. Tumour-infiltrating lymphocytes in melanoma prognosis and cancer immunotherapy. Pathology 2016;48(2):177-87.

17. Burton $A L$, Roach $B A$, Mays $M P$, Chen $A F$, Ginter $B A$, Vierling AM, Scoggins CR, Martin RC, Stromberg AJ, Hagendoorn $L$, McMasters KM. Prognostic significance of tumor infiltrating lymphocytes in melanoma. Am Surg 2011;77(2):188-92.

18. Barnhill RL, Fine JA, Roush GC, Berwick M. Predicting five-year outcome for patients with cutaneous melanoma in a population-based study. Cancer 1996;78(3):427-32.

19. Taylor RC, Patel A, Panageas KS, Busam KJ, Brady MS. Tumorinfiltrating lymphocytes predict sentinel lymph node positivity in patients with cutaneous melanoma. J Clin Oncol 2007;25(7):869-75.

20. Koch M, Beckhove $P$, op den Winkel J, Autenrieth $D$, Wagner $P$, Nummer D, Specht S, Antolovic D, Galindo L, Schmitz-Winnenthal $\mathrm{FH}$, Schirrmacher V, Büchle MWr, Weitz J.Tumor infiltrating T lymphocytes in colorectal cancer: Tumor-selective activation and cytotoxic activity in situ. Ann Surg 2006;244:986-92.

21. Zidlik V, Brychtova S, Uvirova M, Ziak D, Dvorackova J. The changes of angiogenesis and immune cell infiltration in the intra- and peri-tumoral melanoma microenvironment. Int J Mol Sci 2015;16(4):787689.

22. Oble DA, Loewe R, Yu P, Minhm MC Jr. Focus on TILs: prognostic significance of tumor infiltrating lymphocytes in human melanoma. Cancer Immun 2009;9:3.

23. Larosa DF, Orange JS. Lymphocytes. J Allergy Clin Immunol 2008;121(2 Suppl):S364-9; quiz S412.

24. de Vries IJ, Castelli C, Huygens C, Jacobs JF, Stockis J, Schuler-Thurner B, Adema GJ, Punt CJ, Rivoltini L, Schuler G, Coulie PG, Lucas S. Frequency of circulating Tregs with demethylated FOXP3 Intron 1 in Melanoma Patients Receiving Tumor Vaccines and Potentially Treg-depleting Agents. Clin Cancer Res 2011;17(4):841-48.

25. Zhu J, Thakolwiboon S, Liu X, Zhang M, Lubman DM. Overexpression of CD90 (Thy-1) in Pancreatic Adenocarcinoma present in the Tumor Microenvironment. PLoS One 2014;9(12):e115507.

26. Biedermann T, Röcken M, Carballido JM. TH1 and TH2 lymphocytes development and regulation of TH cell - mediated immune responses of the skin. J Investig Dermatol Symp Proc 2004;9(1):5-14.

27. Luckheeram RV, Zhou R, Verma AD, Xia B. CD4+T cells: Differentiation and functions. Clin Dev Immunol 2012;2012:925135.

28. Gandini S, Massi D, Mandalà M. PD-L1 expression in cancer patients receiving anti PD-1/PD-L1 antibodies: A systematic review and metaanalysis. Crit Rev Oncol Hematol 2016;100:88-98.

29. Floess S, Freyer J, Siewert C, Baron U, Olek S, Polansky J, Schlawe K, et al. Epigenetic control of the foxp3 locus in regulatory $T$ cells. PLoS Biol 2007;5(2):e38.

30. Wang Z, Liu JQ, Liu Z, Shen R, Zhang G, Xu J, Basu S, Feng Y, Bai XF. Tumor-derived IL-35 promotes tumor growth by enhancing myeloid cell accumulation and angiogenesis. J Immunol 2013;190(5):241523.

31. Wilke MC, Wu K, Zhao E, Wang G, Zou W. Prognostic Significance of regulatory T cells in tumor. Int J Cancer 2010;127(4):748-58.

32. Roncador G, Brown PJ, Maestre L, Hue S, Martínez-Torrecuadrada JL, Ling KL, Pratap S, Toms C, Fox BC, Cerundolo V, Powrie F, Banham $\mathrm{AH}$. Analysis of FOXP3 protein expression in human CD4+CD25+ regulatory T cells at the single-cell level. Eur J Immunol 2005;35(6):168191.

33. Apostolou I, Verginis $P$, Kretschmer K, Polansky J, Hühn J, von Boehmer H. Peripherally induced Treg: mode, stability, and role in specific tolerance. J Clin Immunol 2008;28:619-24. 
34. Lucas S, van Baren N, de Smet C, Coulie PG. Demethylation of the FOXP3 gene in human melanoma cells preclude the use of this epigenetic mark for quantification of Tregs in unseparated melanoma samples. Int J Cancer 2012;130(8):1960-6.

35. Fu S, Zhang N, Yopp AC, Chen D, Mao M, Chen D, Zhang $H, T G F-\beta$ Induces FOXP3+ T-regulatory Cells from CD4+CD25- Precursors. Am J Transplant 2004;4(10):1614-27.

36. Niu J, Jiang CH, Li CH, Liu L, Li K. Jian Z, Gau T. Foxp3 expression in melanoma cells as a possible mechanism of resistance to immune destruction. Cancer Immunol Immunother 2011;60:1109-18.

37. Schubert K, Gutknecht D, Köberle M, Anderegg U, Saalbach A Melanoma Cells Use Thy-1 (CD90) on Endothelial Cells for Metastasis Formation. Am J Pathol 2013;182(1):66-276.

38. Ma MW, Medicherla RC, Qian M, Vega-Saenz de Miera E, Friedman EB, Berman RS, Shapiro RL, Pavlick AC, Ott PA, Bhardwaj N, Shao Y, Osman I, Darvishian F. Immune response in melanoma: an in-depth analysis of the primary tumor and corresponding sentinel lymph node. Mod Pathol 2012;25(7):1000-10.

39. Sakaguchi S, Wing K, Onishi Y, Prieto-Martin P, Yamaguchi T. Regulatory T cells: how do they suppress immune responses? Int Immunol 2009;21(10):1105-11.

40. Hall BM, Verma ND, Tran GT, Hodgkinson SJ. Distinct regulatory CD4+ T cell subsets; differences between naïve and antigen specific T regulatory cells. Curr Opin Immunol 2011;23(5):641-7.

41. Chen W, Konkel JE. TGF- $\beta$ and "adaptive" Foxp3+ regulatory T cells. J Mol Cell Biol 2010;2(1):30-6.

42. Skarmoutsou E, Bevelacqua V, D’Amico F, Russo A, Spandidos AD Scalisi A, Malaponte G, Guarneri C. FOXP3 expression is modulated by TGF- $\beta 1 / N O T C H 1$ pathway in human melanoma. Int J Mol Med 2018:42(1):392-404.

43. Baumgartner J, Wilson C, Palmer B, Richter D, Banerjee A, McCarter M. Melanoma Induces Immunosuppression by Upregulating FOXP3+ Regulatory T Cells. J Surg Res 2007;141(1):72-7.

44. Wang $X$, Cui $Y$, Luo G, Wang Q, Hu J, He W, Yuan J, Zhou J, Wu Y, Sun X, Robson SC, Li X, Tan J, Peng Y, Xue G, Lu L, Gao W, Wu J. Activated mouse CD4(+)Foxp3(-) T cells facilitate melanoma metastasis via Qa-1-dependent suppression of NK-cell cytotoxicity. Cell Res 2012;22(12):1696-706.

45. Prall F, Dührkop T, Weirich V, Ostwald C, Lenz P, Nizze H, Barten M Prognostic role of CD8+ tumor-infiltrating lymphocytes in stage II colorectal cancer with and without microsatellite instability. Hum Pathol 2004;35(7):808-16.
46. Pardoll DM. The blockade of immune checkpoints in cancer immunotherapy. Nat Rev Cancer 2012;12(4):252-64.

47. Kim HR, Ha SJ, Hong MH, Heo SJ, Koh YW, Choi EC, Kim EK, Pyo KH, Jung I, Seo D, Choi J, Cho BC, Yoon SO. PD-L1 expression on immune cells, but not on tumor cells, is a favorable prognostic factor for head and neck cancer patients. Sci Rep 2016;6:36956.

48. Kakavand $H$, Wilmott JS, Menzies AM, Vilain R, Haydu LE, Yearley JH, Thompson JF, Kefford RF, Hersey P, Long GV, Scolyer RA. PD-L1 expression and tumor-infiltrating lymphocytes define different subsets of MAPK inhibitor-treated melanoma patients. Clin Cancer Res 2015;21(14):3140-8.

49. Ribas A, Shin DS, Zaretsky J, Frederiksen J, Cornish A, Avramis E, Seja E, Kivork C, Siebert J, Kaplan-Lefko P, Wang X, Chmielowski B, Glaspy JA, Tumeh PC, Chodon T, Pe'er D, Comin-Anduix B. PD-1 Blockade Expands Intratumoral Memory T Cells. Cancer Immunol Res 2016;4(3):194-203.

50. Li Y, Liang L, Dai W, Cai G, Xu Y, Li X, Li Q, Cai S. Prognostic impact of programmed cell death-1 (PD-1) and PD-ligand 1 (PD-L1) expression in cancer cells and tumor infiltrating lymphocytes in colorectal cancer. Mol Cancer 2016;15(1):55

51. Phillips T, Simmons P, Inzunza HD, Cogswell J, Novotny J Jr, Taylor C, Zhang X. Development of an automated PD-L1 immunohistochemistry (IHC) assay for non-small cell lung cancer. Appl Immunohistochem Mol Morphol 2015;23(8):541-9.

52. Simon S, Labarriere N. PD-1 expression on tumor-specific T cells: Friend or foe for immunotherapy? Oncoimmunology 2017;7(1):e1364828.

53. Inozume T, Hanada K, Wang QJ, Ahmadzadeh M, Wunderlich JR, Rosenberg SA, Yang JC. Selection of CD8+PD-1+ lymphocytes in fresh human melanomas enriches for tumor-reactive T cells. J Immunother 2010;33(9):956-64.

54. Gros A, Robbins PF, Yao X, Li YF, Turcotte S, Tran E, Wunderlich JR, Mixon A, Farid S, Dudley ME, Hanada K, Almeida JR, Darko S, Douek DC, Yang JC, Rosenberg SA. PD-1 identifies the patient-specific CD8 ${ }^{+}$tumor-reactive repertoire infiltrating human tumors. J Clin Invest 2014;124(5):2246-59.

55. Ott PA, Hodi FS, Robert C. CTLA-4 and PD-1/PD-L1 blockade: new immunotherapeutic modalities with durable clinicalbenefit in melanoma patients. Clin Cancer Res 2013;19(19):5300-9. L1 blockade: new immunotherapeutic modalities with durable clinicalbenefit in melanoma patients. Clin Cancer Res 2013;19(19):5300-9. 